\title{
Correction to: The vasculature niches required for hematopoiesis
}

\author{
Zuohua Chi ${ }^{1} \cdot$ Liji Chen $^{2} \cdot$ Xiaomin $\mathrm{Ye}^{2} \cdot$ Aimei Liu $^{2} \cdot$ Gongwang Yu ${ }^{2} \cdot$ Yan Sun Si, $^{2,4}$
}

Published online: 24 November 2021

(c) Springer-Verlag GmbH Germany, part of Springer Nature 2021

\section{Correction to: Journal of Molecular Medicine} https://doi.org/10.1007/s00109-021-02155-2

The image of Fig. 1 is modified in the published proof. In the modified image of Fig. 1, the missing " $-1 "$ is added and "CD31+Sca-VEGFR2+VEGFR3+ cells (sBMECs)" is corrected to "CD31+Sca-1-VEGFR2+VEGFR3+ cells (sBMECs)". In addition, the image of Fig. 1 with improved quality and resolution is provided. The correct Fig. 1 is shown below.

The Original article has been corrected.

The original article can be found online at https://doi.org/10.1007/ s00109-021-02155-2.

Yan Sun

sxmshw77@163.com

1 The First Affiliated Hospital/School of Clinical Medicine, Guangdong Pharmaceutical University, Guangzhou, China

2 College of Life Science and Biopharmaceutics, Pharmaceutical University, GuangdongGuangzhou, China

3 Guangdong Province Key Laboratory for Biotechnology, Drug Candidates, Guangdong Pharmaceutical University, Guangzhou, China

4 Department of Cellular Biology, Institute of Biomedicine, Key Laboratory of Bioengineering Medicine of Guangdong, Jinan University, ProvinceGuangzhou, Guangdong, China 


\section{Vasculature niches}

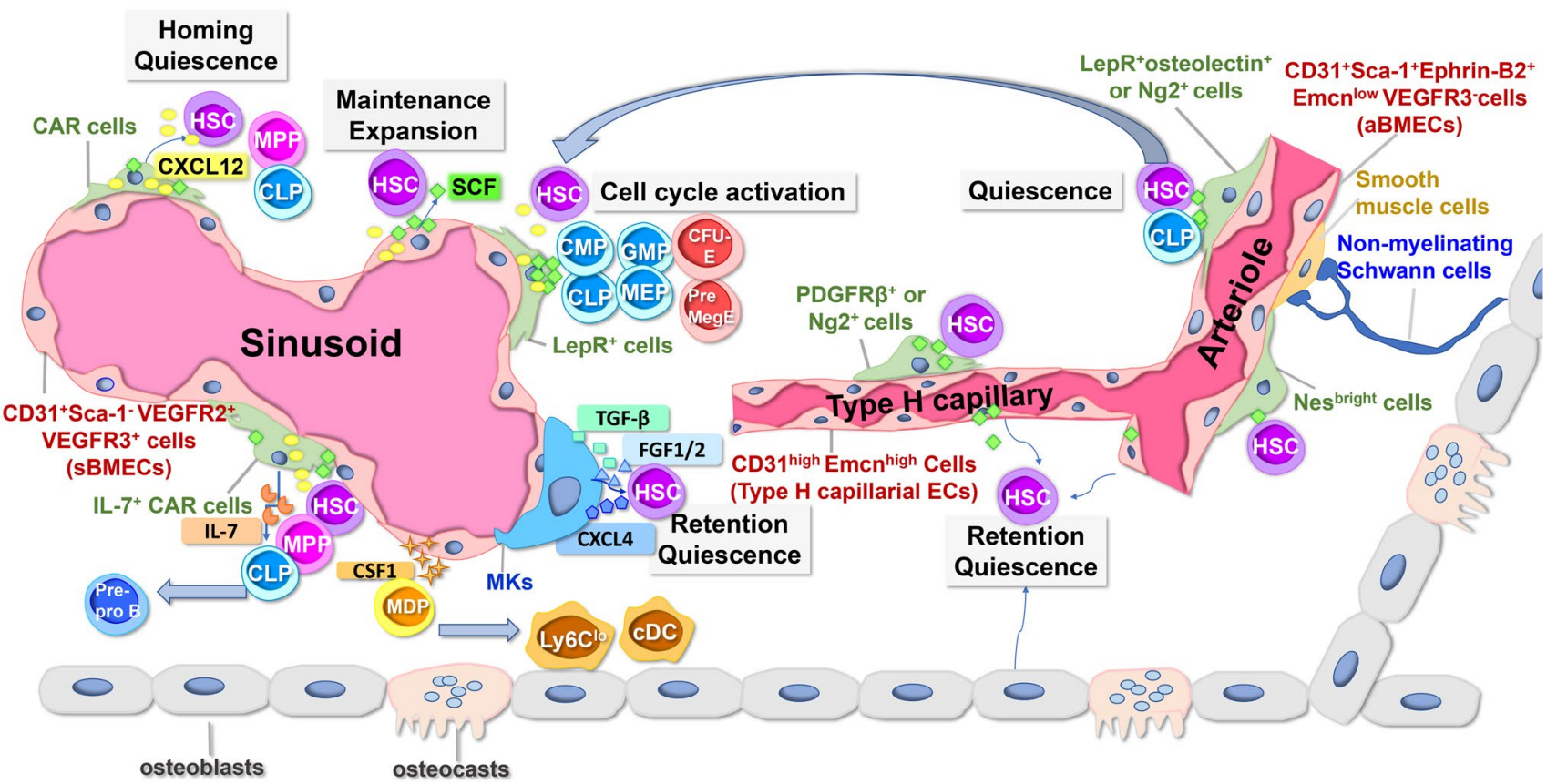

Fig. 11 Anatomical constituents and function of VNs in the BM

Publisher's Note Springer Nature remains neutral with regard to jurisdictional claims in published maps and institutional affiliations. 